\title{
Imaging of structures in the high-latitude ionosphere: model comparisons
}

\author{
D. W. Idenden ${ }^{1}$, R. J. Moffett ${ }^{1}$, M. J. Williams ${ }^{2}$, P. S. J. Spencer ${ }^{2}$, L. Kersley ${ }^{2}$ \\ ${ }^{1}$ School of Mathematics and Statistics, University of Sheffield, The Hicks Building, Sheffield, S3 7RH, UK \\ ${ }^{2}$ Department of Physics, University of Wales, Aberystwyth, SY23 3BZ, UK
}

Received: 15 September 1997 / Revised: 10 April 1998 / Accepted: 14 April 1998

\begin{abstract}
The tomographic reconstruction technique generates a two-dimensional latitude versus height electron density distribution from sets of slant total electron content measurements (TEC) along ray paths between beacon satellites and ground-based radio receivers. In this note, the technique is applied to TEC values obtained from data simulated by the Sheffield/ UCL/SEL Coupled Thermosphere/Ionosphere/Model (CTIM). A comparison of the resulting reconstructed image with the 'input' modelled data allows for verification of the reconstruction technique. All the features of the high-latitude ionosphere in the model data are reproduced well in the tomographic image. Reconstructed vertical TEC values follow closely the modelled values, with the F-layer maximum density $(\mathrm{NmF} 2)$ agreeing generally within about $10 \%$. The method has also been able successfully to reproduce underlying auroral-E ionisation over a restricted latitudinal range in part of the image. The height of the F2 peak is generally in agreement to within about the vertical image resolution $(25 \mathrm{~km})$.
\end{abstract}

Key words. Ionosphere (modelling and forecasting; polar ionosphere) · Radio Science (instruments and techniques)

\section{Introduction}

Significant progress has been made since the early 1990s in the development of radio tomography as a new experimental technique for the study of the spatial distribution of the electron density in the ionised atmosphere (Kersley and Pryse, 1994). Independent verification of the method has been obtained at auroral

Correspondence to: D. W. Idenden latitudes from observations using the EISCAT incoherent scatter radar (Kersley et al., 1993; Walker et al., 1996). It is in the hostile environment of the highest latitudes that ionospheric tomography offers considerable potential, with its ability to image spatial structures over a wide region from a limited number of ground stations. The present work reports results from a study of the use of tomography in the high-latitude ionosphere in which independent verification has been obtained using the Sheffield/UCL/SEL Coupled Thermosphere/ Ionosphere/Model (Fuller-Rowell et al., 1996).

\section{Ionospheric tomography}

Ionospheric tomography uses radio signals from satellites received at a chain of stations approximately aligned in the sub-satellite track. Each receiver provides a sequence of measurements of total electron content (TEC) with a constant unknown offset along the receiver-satellite ray path. Thus, a large number of relative TEC measurements along intersecting ray paths are obtained. These are inverted in a reconstruction algorithm to create a spatial image in two dimensions of the electron density. Transmissions from satellites in the former Navy Navigation Satellite System (NNSS) are used in ionsopheric tomography, these satellites being in near circular polar orbits at altitudes of about $1100 \mathrm{~km}$. A number of different reconstruction methods have been used in ionospheric tomography, with much effort directed to minimising the limitations imposed by the lack of horizontal ray paths in satellite-to-ground geometry.

The reconstruction technique for the present work uses a quadratic programming $(\mathrm{QP})$ approach, (Spencer, 1998). QP methods involve solving linear equations such that a quadratic objective function relating the unknown is minimised. In the current application the constant TEC offsets are eliminated by taking differences in TECs between neighbouring ray paths. Difference ray-path line integrals are then solved for a pixel-based representation 
of the ionosphere, such that the electron density within each pixel is positive. Stable reconstructions are ensured by solving simultaneously for a continuous background ionosphere, defined by the linear sum of a number of functions derived from Chapman profiles. By using the objective function to minimise the difference between the two representations, stable solutions are obtained over a range of peak heights and profile shapes. This technique also ensures physically realistic solutions, and produces absolute, not relative, electron densities.

The receiver sites chosen for this simulation are the six locations in Scandinavia that have been used on a campaign basis by the Aberystwyth group for experimental studies of the high-latitude ionosphere using the tomographic method. The coordinates of the stations are listed in Table 1.

\section{CTIM model description}

The tomographic reconstruction technique was applied to slant TECs, with an unknown constant offset, calculated from data obtained using results from the Sheffield/UCL/SEL Coupled Thermosphere/Ionosphere/Model (CTIM) (Fuller-Rowell et al., 1996). It should be noted that relative, that is not absolute, TEC values form the input to the reconstruction algorithm used in the present study. Only the essential features of the CTIM model relevant to the present work are given here. More detailed descriptions can be found in the aforementioned reference. Model electron densities are calculated in the semi-Lagrangian (Fuller-Rowell et al., 1987 ; 1988) high-latitude ionosphere code that solves the coupled equations of continuity and momentum on straight 'open' field lines for the major species in the Flayer and topside, $\mathrm{O}^{+}$and $\mathrm{H}^{+}$, poleward of $\pm 35^{\circ}$ latitude. The field lines extend from an altitude of $100 \mathrm{~km}$ to $10000 \mathrm{~km}$ and are fixed in longitude and latitude, with separations of $18^{\circ}$ and $2^{\circ}$ respectively. In the present work, the input $\mathbf{E} \times \mathbf{B}$, drifts and precipitation patterns come from the empirical models of Foster et al. (1986) and Fuller-Rowell and Evans (1987) respectively. These models are each parametrised by the hemisphere power index based on TIROS/NOAA precipitating particle measurements and are mutually consistent. The boundary conditions of chemical equilibrium at the bottom and zero flux at the top of the fluxtable are imposed for the $\mathrm{O}^{+}$ion. While chemical equilibrium at $100 \mathrm{~km}$ is also assumed for $\mathrm{H}^{+}$, account is taken of the polar wind by imposing an empirically-

Table 1. Geographic coordinates of satellite receiving stations

\begin{tabular}{lll}
\hline & Latitude $\left(^{\circ}\right)$ & Longitude $\left(^{\circ}\right)$ \\
\hline Uppsala & 59.80 & 17.60 \\
Lycksele & 64.62 & 18.76 \\
Kiruna & 67.90 & 20.40 \\
Tromso & 69.60 & 19.20 \\
Longyearbyen & 78.15 & 15.30 \\
Ny Alesund & 78.90 & 12.00 \\
\hline
\end{tabular}

based flux at $10000 \mathrm{~km}$ (Allen et al., 1986). The concentrations of the molecular ions, $\mathrm{N}_{2}{ }^{+}, \mathrm{O}_{2}{ }^{+}$and $\mathrm{NO}^{+}$, and the atomic ion $\mathrm{N}^{+}$, are calculated by assuming chemical equilibrium. Ion temperatures are computed by solving the energy balance equation, which assumes that the rate at which heat is gained from the electron gas and by frictional heating is equal to the rate at which it is lost to the neutral air.

The thermospheric code solves the coupled momentum, continuity and energy equations by an explicit numerical scheme. Neutral temperatures, winds and densities are calculated on a global grid with the same horizontal resolution as the ionospheric code and with 15 fixed pressure levels between altitudes of $80 \mathrm{~km}$ and 400 to $600 \mathrm{~km}$ depending on conditions. The calculation of coupling terms such as the ion drag force (Lorentz force), and Joule heating require ionospheric densities and conductivities. Poleward of $\pm 35^{\circ}$ these are supplied by the high-latitude ionospheric code, Elsewhere the values are provided by the empirical model of Chiu (1975). Conversely, in the high-latitude code, calculations of the heat exchange with the neutral gas, ionneutral frictional heating, field aligned ion-neutral momentum transfer, and chemical production and loss rates require thermospheric temperatures, composition, densities, and winds. These parameters are provided by the thermospheric code. Thus, the thermospheric and ionospheric codes are fully coupled at high latitudes.

\section{Model starting conditions and outputs}

Model electron densities were calculated for the December solstice (day 355) and convection and precipitation patterns keyed to a hemispheric power index of 6 , corresponding to a $\mathrm{K}_{\mathrm{P}}$ of $3-$. The starting conditions for the simulated satellite pass were obtained initially by running the model under steady-state conditions until the results become diurnally reproducible, which in this case was after four days. The simulated satellite pass began when the satellite was at latitude of $30^{\circ}$ on the $15^{\circ}$ meridian at 19:06 UT. The satellite was assumed to be travelling northwards in a polar orbit at an altitude of $1100 \mathrm{~km}$, typical of the Navy Navigational Satellite System (NNSS) satellites used to obtain slant TECs experimentally. Model electron densities were integrated above $100 \mathrm{~km}$ along ray paths between the satellite and each receiving station (Table 1) from which the satellite would be visible. This set of slant TECs obtained from model data were written to a file of identical format to that obtained experimentally from a real satellite pass and used as input to the tomographic reconstruction algorithm. The path of the simulated satellite pass is shown in Fig. 1, superimposed on a contour plot of model NmF2 values at 19:18 UT, midway through the pass. Also shown are the positions of the northernmost and southernmost stations in the receiving chain.

The electron densities at the layer peak illustrate features that are characteristic of the high-latitude ionosphere in winter. The tongue of dayside ionisation from sunlit lower latitudes can be seen feeding into the 


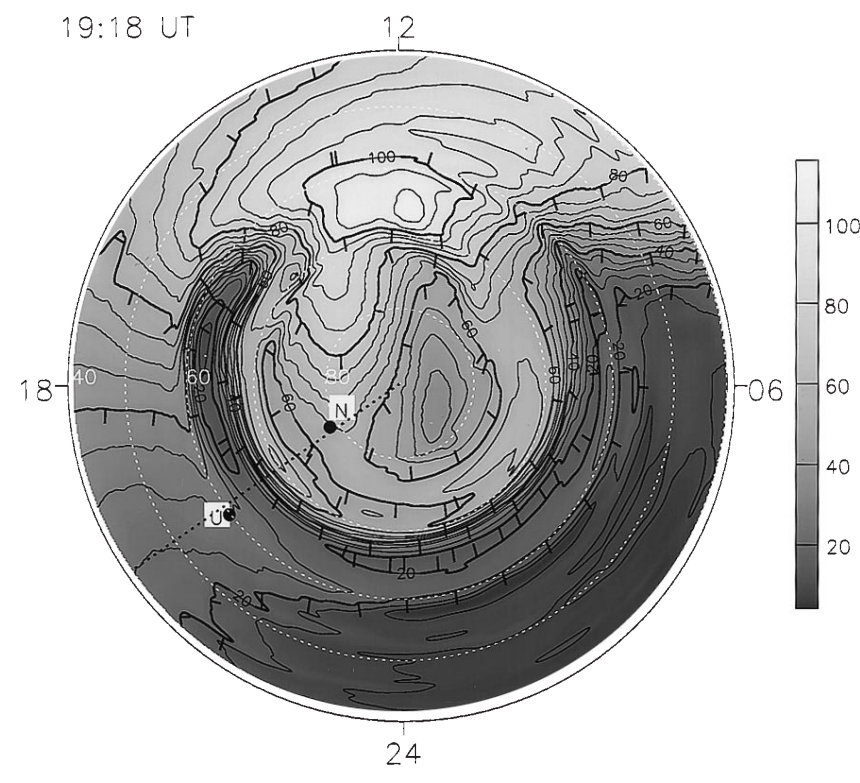

Fig. 1. Northern Hemisphere polar contour plot of modelled winter solstice $\mathrm{NmF} 2$ values extending down to $40^{\circ}$ latitude. Values on the bold contours are in units of $10^{10} \mathrm{~m}^{-3}$. Contours are separated by $5 \times 10^{10} \mathrm{~m}^{-3}$. The tick marks on the bold contours show the direction in which $\mathrm{NmF} 2$ decreases. The dotted black line shows the simulated satellite path. Also shown are the positions of the southermost and northernmost receiving stations at Uppsala $(U)$ and $\mathrm{Ny}$ Alesund $(N)$ respectively

polar cap in the post-noon sector. The polar hole is also clearly evident, where flux tubes circulate in winter darkness trapped in the dawn convection cell, though the geometry of the present pass does not intersect this region. A narrow band of enhanced density can be seen at auroral latitudes in the evening sector, with a steep wall leading into the main trough on the equatorwards side also clearly visible.

\section{Results}

The tomographic reconstruction technique was applied to the data set of TEC values obtained from the model results as described. The modelled and reconstructed electron densities are shown as contour plots, in a latitude versus height plane, in Fig. 2a,b respectively. It can be seen that all of the major features are reproduced in the tomographic image. These can be most easily identified by reference to the polar plot in Fig. 1. The tongue of ionisation feeding from the dayside across the polar cap in the convective flow can be identified with the maximum in the image at about $80^{\circ} \mathrm{N}$ latitude. The auroral zone, with a boundary blob surmounting the poleward wall of the trough, and underlying auroral-E layer can also be seen. The trough itself, with steep poleward wall and shallower gradient on the equatorward side leading to the mid-latitude ionosphere, has also been reproduced in the image. The overall impression gained from comparison of image and model is that the horizontal features are well replicated. In the vertical, the gradient of the bottomside appears mar-
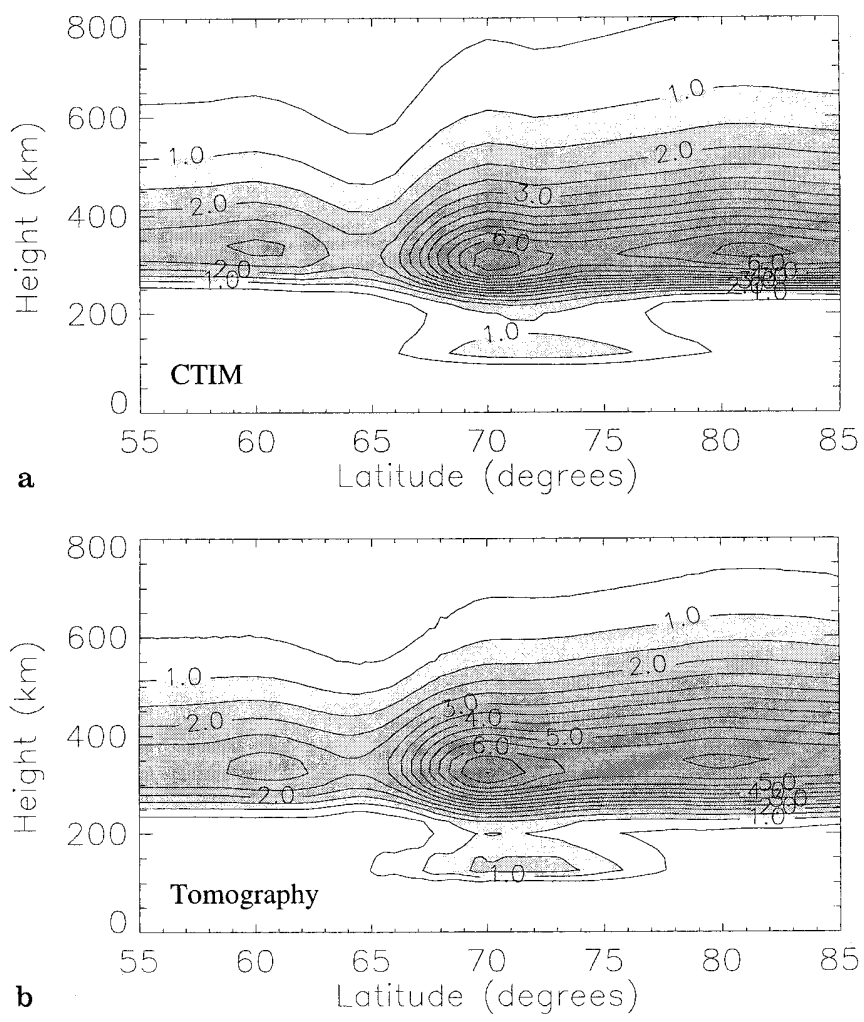

Fig. 2a, b. Contour plots of a modelled and $\mathbf{b}$ reconstructed electron density distributions for the altitude/latitude slice below the simulated satellite track at $15^{\circ} \mathrm{E}$ longitude. Contour values are in units of $10^{10} \mathrm{~m}^{-3}$. The positions of the mid-latitude trough, the auroral region and the polar cap tongue of ionisation can be identified

ginally less steep than in the original, while the topside scale height differs slightly, causing the densities at the layer peak in the boundary blob and tongue of ionisation to be a little greater than those in the model. The tongue of ionisation, therefore, is a little less welldefined in the image.

More objective comparisons have been made in Fig. $3 a-c$, where the vertical TEC, the density at the layer peak $(\mathrm{NmF} 2)$ and the height of the $\mathrm{F} 2$ peak $(\mathrm{hmF} 2)$ are plotted as functions of latitude, for both modelled and reconstructed values. It can be seen that the tomographic technique gives vertical TEC values that are accurate to much better than $10 \%$ in general. This agreement is excellent bearing in mind that relative values of slant TEC were used as input to the reconstruction algorithm. The reconstructed NmF2 values show good agreement with the originals, but overestimate the densities in the trough minimum, and through the poleward wall leading to the boundary blob. By contrast, in the region of the tongue of ionisation the peak density in the image is some $10 \%$ below that of the model and it is in this region that the poorest agreement is found. The positions of the minimum and maximum associated with the trough are well within the one degree resolution of the model. The image was created on a $0.25^{\circ}$ latitudinal grid. The values of $\mathrm{hmF} 2$ have been estimated using a curve-fitting technique through the pixels about the layer peak. The agreement is within \pm one pixel height for both 

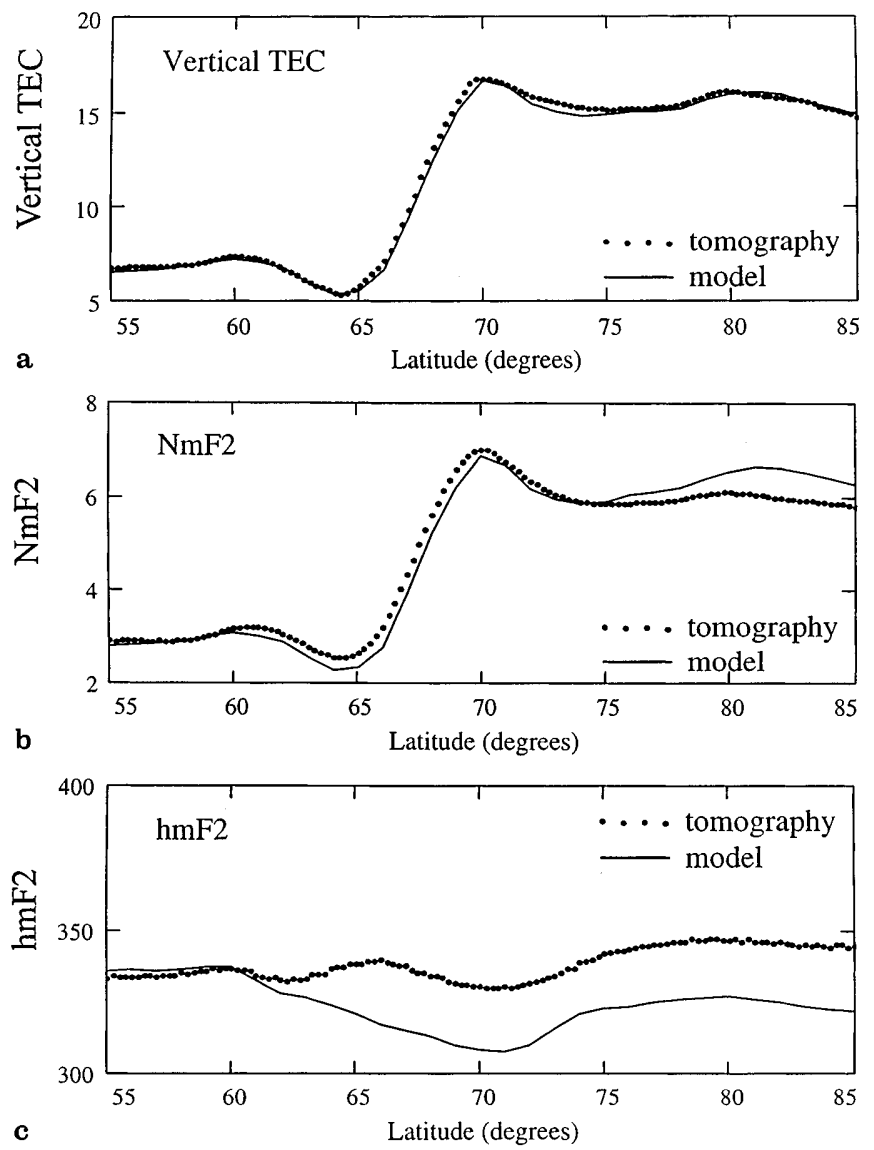

Fig. 3a-c. Comparisons of modelled (solid) and reconstructed (dotted) a vertical TEC $\left(\times 10^{16} \mathrm{~m}^{-2}\right), \mathbf{b}\left(\times 10^{11} \mathrm{~m}^{-3}\right) \mathrm{NmF} 2$ and $\mathbf{c} \mathrm{hmF} 2(\mathrm{~km})$ values

reconstruction $(25 \mathrm{~km})$ and model $(20 \mathrm{~km})$ throughout the latitudinal range, though the image height exceeds that of the model everywhere apart from in the midlatitude section. However, it can be noted that, apart from in the trough minimum itself, the form of the height change of the layer peak is followed in the image.

A comparison of the vertical profile shapes from both model and image, at three latitudes, is shown in Fig. $4 \mathrm{a}-$ c. In general, there is very good treatment, though the densities in the high topside are consistently underestimated by a small amount throughout the entire latitudinal range of the reconstruction. At $60^{\circ} \mathrm{N}$ the peak density in the image is about $5 \%$ too large, though the peak heights show good agreement. Differences in the profiles at $70^{\circ} \mathrm{N}$ can be attributed largely to the slight discrepancy in the peak height, though the tomographic technique has overestimated the peak density by a few percent. The auroral-E layer has been reproduced in the image, though its density is larger than in the model. At $80^{\circ} \mathrm{N}$ the main difference is in the density at the peak, though the discrepancy is still less than $10 \%$.

\section{Discussion}

The work described has demonstrated that the tomographic reconstruction technique is highly effective at

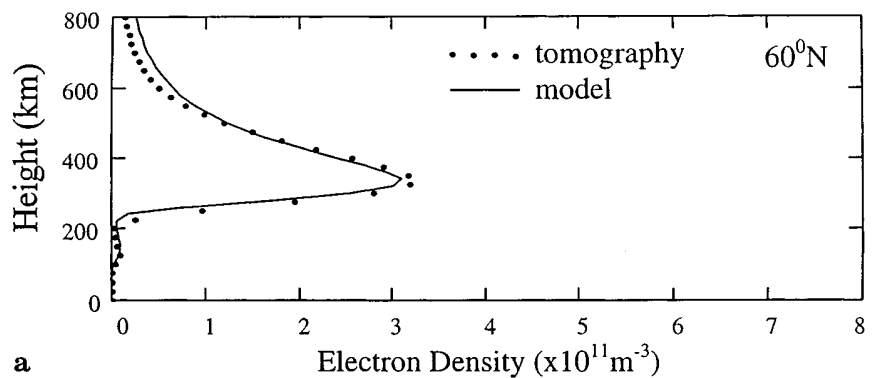

a
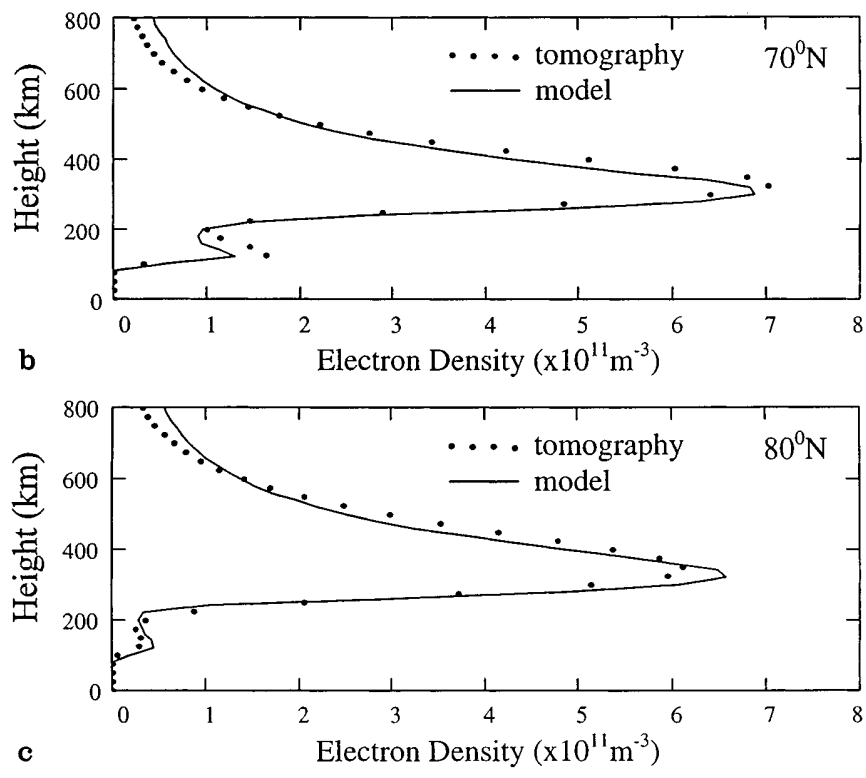

Fig. 4a-c. Comparisons of modelled (solid lines) and reconstructed (dotted lines) altitude profiles of electron density at latitudes of $\mathbf{a} 60^{\circ} \mathrm{N}$, b $70^{\circ} \mathrm{N}$ and c $80^{\circ} \mathrm{N}$

imaging ionospheric features at high latitudes. The method replicates well the horizontal structures in the ionisation, though the lack of horizontal ray paths in satellite-to-ground geometry results in greater uncertainty in the imaging of the vertical profile. The present results show that the quadratic programming method used here has been able to reproduce most of the essential features of the vertical structure of the ionisation, including the situation when there is an underlying auroral-E layer over only a limited latitudinal extent of the image. The discrepancies in the densities around the F2 layer peak are generally less than $10 \%$, while differences in peak height are close to the limit set by the pixel resolution. The departures in profile shape in the high topside between model input and the reconstructed image can be attributed to the limitation imposed on the current work by the use in the quadratic programming method of only Chapman-type profiles. Modifications to the method to incorporate a wider range of possible vertical ionospheric profiles in the high topside could result in better agreement in that region. Improvements in the reproduction of the density at the peak of the layer would also be expected from this change. In essence, the tomographic method redistributes the line integral of the density, the total electron content, throughout the profile, so that small discrep- 
ancies in topside shape are compensated by more noticeable changes in peak density. While there are still improvements to be made, the present work shows that ionospheric tomography has advanced to a stage where it can yield reliable reproductions of the essential broad features of the high-latitude ionosphere.

Acknowledgements. The work was supported by PPARC awards GR/K06112 and GR/K98797 at Sheffield and Aberystwyth respectively. MJW acknowledges the support of PPARC research studentship.

Topical Editor D. Alcaydé thanks D. Rees for his help in evaluating this paper.

\section{References}

Allen, B. T., G. J. Bailey, and R. J. Moffett, Ion distributions in the high-latitude topside ionosphere, Ann. Geophysicae, 4, 97, 1986.

Chiu, Y.T., An improved phenomenological model of ionospheric density, J. Atmos. Terr. Phys., 37, 1563, 1975.

Foster, J. C., J. M. Holt, R. G. Musgrove, and D. S. Evans, Ionspheric convection associated with discrete levels of particle precipitation, Geophys. Res. Lett., 13, 656, 1986.

Fuller-Rowell, T. J., and D. S. Evans, Height integrated Pederson and Hall conductivity patterns inferred from the TIROSNOAA satellite data, J. Geophys. Res., 92, 7606, 1987.
Fuller-Rowell, T. J., D. Rees, S. Quegan, R. J. Moffett, and G. J. Bailey, Interactions between neutral thermospheric composition and the polar ionisation using a coupled ionosphere-thermosphere model, J. Geophys. Res., 92, 7744, 1987.

Fuller-Rowell, T. J., D. Rees, S. Quegan, R. J. Moffett, and G. J. Bailey, Simulations of the seasonal and universal time variations of the thermosphere and ionosphere using a coupled, three-dimensional, global model, Pure Appl. Geophys., 127, 189, 1988.

Fuller-Rowell, T. J., D. Rees, S. Quegan, R. J. Moffett, M. V. Codrescu, and G. H. Millward, A coupled thermosphereionosphere model (CTIM), in STEP Handbook on Ionospheric Models, Ed. R. W. Schunk, 217, 1996.

Kersley, L., and S. E. Pryse, Development of experimental ionospheric tomography, Int. J. Imag. Syst. Tecn., 5, 141, 1994.

Kersley, L., J. A. T. Heaton, S. E. Pryse, and T. D. Raymund, Experimental ionospheric tomography with ionsonde input and EISCAT verification, Ann. Geophysicae, 11, 1064, 1993.

Spencer, P. S. J., A new solution to the problem of ionospheric tomography using quadratic programming, Radio Sci. (in press), 1998.

Walker, I. K., J. A. T. Heaton, L. Kersley, C. N. Mitchell, S. E. Pryse, and M. J. Williams, EISCAT verification in the development of ionospheric tomography, Ann. Geophysicae, 14, 1413, 1996. 